\title{
Predicción de la Composición Química y Fibra Detergente Neu- tro de Rye Grass Italiano (Lolium multiflorum Lam) mediante Espectroscopía de Reflectancia en Infrarrojo Cercano (NIRS)
}

\author{
Prediction of Chemical Composition and Neutral Detergent Fibre of Italian \\ Ryegrass (Lolium multiflorum LAM) by Near INFRAREd SPeCtroscopy (NIRS)
}

Sandra Bezada Q., ${ }^{1,6}$, Teresa Arbaiza F. ${ }^{1}$, Fernando Carcelén C. ${ }^{1}$, Felipe San Martín H. ${ }^{1}$, Christian López L. ${ }^{2}$, Jean Rojas E. ${ }^{3}$, Virginia Rivadeneira ${ }^{1}$, Oscar Espezúa F. ${ }^{1}$, Jorge Guevara V. ${ }^{4}$, Víctor Vélez $M^{5}{ }^{5}$

\section{Resumen}

El objetivo del presente estudio fue generar ecuaciones de calibración que permitan predecir la composición químico nutricional de la especie forrajera rye grass italiano (RG) (Lolium multiflorum Lam) mediante la técnica de Espectroscopía de Reflectancia en Infrarrojo Cercano (NIRS). Se colectaron 75 muestras de RG de diferentes semanas de corte provenientes de los campos experimentales del Centro de Investigacion IVITA-El Mantaro (Huancayo, Perú), a las cuales se les realizó la captura del espectro mediante equipo NIRS y se hizo el análisis químico de referencia para los componentes proteína cruda (PC), extracto etéreo (EE), cenizas totales (CZ), fibra cruda (FC) y fibra detergente neutro (FDN). Se desarrolló un modelo de calibración y validación mediante mínimos cuadrados parciales (PLS) y como estadísticos de exactitud y precisión se utilizaron el coeficiente de correlación $(\mathrm{R})$, coeficiente de determinación $\left(\mathrm{R}^{2}\right)$, raíz cuadrada media del error de calibración (RMSEC), raíz cuadrada media del error de predicción (RMSEP), proporción del rango con el error (RER) y desviación residual predictiva (RPD). El análisis proximal promedio fue para $\mathrm{PC}=19.02 \%, \mathrm{EE}=4.53 \%, \mathrm{CZ}=12.79 \%, \mathrm{FC}=16.50 \%$ y $\mathrm{FDN}=60.98 \%$. Altos valores de $\mathrm{R}^{2} \mathrm{y}$ bajos RMSEC y RMSEP fueron obtenidos para PC

\footnotetext{
${ }^{1}$ Laboratorio de Bioquímica, Nutrición y Alimentación Animal, Facultad de Medicina Veterinaria, Universidad Nacional Mayor de San Marcos, Lima, Perú

${ }^{2}$ Reactivos para Análisis Perú SA, Lima, Perú

${ }^{3}$ Estación Experimental del Centro de Investigación IVITA-El Mantaro, Universidad Nacional Mayor de San Marcos, Huancayo, Perú

${ }^{4}$ EAP Ingeniería Agroindustrial, Facultad de Química e Ingeniería Química, Universidad Nacional Mayor de San Marcos, Lima, Perú

${ }^{5}$ Estación Experimental del Centro de Investigación IVITA-Maranganí, Universidad Nacional Mayor de San Marcos, Cusco, Perú

${ }^{6}$ E-mail: sandrabezadaq@gmail.com
} 
$(0.96,1.02,1.19), \mathrm{EE}(0.94,0.29,1.05), \mathrm{CZ}(0.90,0.57,0.92)$ y $\mathrm{FDN}(0.90,1.01,1.25$, respectivamente). El mayor RER (22.34) y RPD (4.90) se obtuvo para EE. Se concluye que las ecuaciones de calibración y validación NIRS obtenidas permiten una óptima predicción cuantitativa de PC, EE, CZ y FDN en rye grass italiano (Lolium multiflorum Lam).

Palabras clave: NIRS; espectroscopía de reflectancia en infrarrojo cercano; evaluación de forrajes; análisis proximal; rye grass italiano; Lolium multiflorum Lam; calibración

\section{Abstract}

The aim of this study was to generate calibration equations to predict the nutritional chemical composition of the Italian rye grass (RG) (Lolium multiflorum Lam) by near infrared spectroscopy (NIRS). A total of 75 samples of RG of different harvesting weeks were collected from the IVITA Research Center in Huancayo (Peru). Spectrum capture was performed using NIRS and the chemical analysis was done for reference of the following components: crude protein (CP), ether extract (EE), total ash (CZ), crude fibre (CF) and neutral detergent fibre (NDF). A calibration and validation model by partial least squares (PLS) was developed and the correlation coefficient (R), coefficient of determination $\left(\mathrm{R}^{2}\right)$, root mean square error of calibration (RMSEC), root mean square error of prediction (RMSEP), ratio range with error (RER) and residual predictive deviation (RPD) were used as statistics of accuracy and precision. Proximate analysis means were: $\mathrm{PC}=19.02 \%, \mathrm{EE}=4.53 \%, \mathrm{CZ}=12.79 \%, \mathrm{FC}=16.50 \%$ and $\mathrm{NDF} 60.98 \%$. High values of $\mathrm{R}^{2}$ and low values of RMSEC and RMSEP were obtained for PC $(0.96,1.02,1.19)$, EE (0.94, $0.29,1.05), \mathrm{CZ}(0.90,0.57,0.92)$ and $\mathrm{NDF}(0.90,1.01,1.25$, respectively). The largest RER (22.34) and RPD (4.90) were obtained for EE. It is concluded that the calibration and validation equations obtained by NIRS enable optimal quantitative prediction of PC, EE, $\mathrm{CZ}$ and NDF in Italian rye grass (Lolium multiflorum Lam).

Key words: NIRS; near infrared spectroscopy; forage evaluation; proximate analysis; Italian rye grass; Lolium multiflorum Lam; calibration

\section{INTRODUCCIÓN}

En el Perú existe un limitado desarrollo en la automatización de los métodos convencionales de análisis de alimentos, de modo que se dificulta disponer de información confiable de forma rápida y eficaz sobre el valor nutricional de los pastos utilizados como alimento para el ganado.

La tecnología de espectroscopía de reflectancia en infrarrojo cercano (Near Infrared Spectroscopy, NIRS) se emplea desde la década del setenta como una técnica alternativa a los métodos químicos y químico-biológicos tradicionales, con alto potencial para obtener estimaciones confiables y rápidas de la composición química nutricional de forrajes (Givens et al., 1997; Cozzolino et al., 2003; Decruyenaere, 2009; Andueza et al., 2011; Decruyenaere et al., 2015; Tellado et al., 2015; Asekova et al., 2016), así como de otros productos de muy diversa índole; entre ellos, los de industrias de alimentos, bioquímica, ambiental, farmacéutica y médica (Shenk y Westerhaus, 1994; Dambergs et al. 2006; Magwaza et al., 2016), constituyéndose una técnica no destructiva, rápida, $\mathrm{y}$ de gran precisión y exactitud. 
La técnica está basada en la medición de la intensidad de absorción de luz en el espectro infrarojo (700-2500 nm) de una muestra, que mediante la aplicación de técnicas de calibración multivariadas se relaciona con la composición o alguna propiedad de la misma (FAO, 2011). Ha sido estudiada para la predicción de la composición nutricional (proteína cruda y fibra cruda) de pastos y forrajes en España (García-Ciudad et al., 1993), EEUU (Krähmer et al., 2013), Australia (Woolnough y Foley, 2002) y Colombia (Lascano, 2002; Vásquez et al. 2004), entre otros.

El rye grass italiano (Lolium multiflorum Lam) es una gramínea perenne de rápido crecimiento, que se cultiva desde el nivel de mar hasta los $4200 \mathrm{msnm}$, lo cual condiciona una elevada demanda como alimento forrajero para el ganado (Ordoñez y Bojórquez, 2004; CARE, 2011; INEI, 2012; Bojórquez et al., 2015). El objetivo de la presente investigación fue evaluar la aplicabilidad de la espectroscopía de reflectancia en infrarrojo cercano (NIRS) para la predicción químico nutricional de rye grass italiano (Lolium multiflorum Lam), conocimiento base para formular raciones eficientes basadas en información actualizada.

\section{Materiales y Métodos}

\section{Lugar de Estudio}

El presente trabajo se realizó en el Laboratorio de Bioquímica, Nutrición y Alimentación Animal (LBNAA) de la Facultad de Medicina Veterinaria de la Universidad Nacional Mayor de San Marcos (Lima, Perú). La zona presenta una temperatura anual promedio de $22{ }^{\circ} \mathrm{C}$ y $83 \%$ de humedad relativa.

El LBNAA cuenta con un equipo de NIRS, Antaris II FT-NIR Analyzer THERMO $^{\text {TM }}$ (longitud de onda de 400 a 2500), que analiza materias primas líquidas y sólidas. El equipo cuenta con una computa- dora y cápsulas de cuarzo de $8 \mathrm{~cm}$ de diámetro para escaneo de muestras secas y molidas. El equipo se encuentra bajo condiciones de $54 \%$ de humedad relativa y $18^{\circ} \mathrm{C}$ de temperatura ambiental. El manejo de la base de datos de los espectros se realiza mediante el software TQ Analyst ${ }^{\mathrm{TM}}$.

\section{Muestras}

Se trabajó con 75 muestras representativas de rye grass italiano (Lolium multiflorum Lam), variedades Tama y Winter Star, con un rango apropiado de características nutricionales de interés, obtenidas en forma experimental mediante muestreo aleatorio estratificado con 4, 6, 9, 12 y 18 semanas de corte. Las muestras correspondieron a cosechas realizadas entre febrero y julio de 2015, y tomadas a $5 \mathrm{~cm}$ del suelo (Pereira $e t$ al., 2012), en lotes experimentales de la estación experimental del Centro de Investigación IVITA en El Mantaro (Junín, Perú), a $3320 \mathrm{msnm}$.

Las muestras se utilizaron para la calibración y validación externa del NIRS. Las muestras fueron colocadas en bolsas de papel kraft y llevadas a estufa convencional a una temperatura de $60^{\circ} \mathrm{C}$ por $48 \mathrm{~h}$. Luego se llevaron al LBNAA para ser molidas (molino Thomas Wiley ${ }^{\mathrm{TM}}$ ) y pasadas por malla de $1 \mathrm{~mm}$. Se almacenaron en bolsas de plástico, tipo ziploc, hasta su análisis espectral y químico proximal.

\section{Análisis Proximal}

El análisis químico proximal de las muestras se realizó en el LBNAA, según el protocolo de la AOAC (1990) para las variables nutricionales de proteína cruda (AOAC 976.05), extracto etéreo (AOAC 920.39), fibra cruda (AOAC 978.10) y cenizas totales (AOAC). En el caso de la fibra detergente neutro (FDN), se siguió el protocolo de Van Soest et al. (1991). Los análisis se realizaron por duplicado y se reportaron como porcentaje en base seca. 


\section{Metodología NIRS}

\section{Registro de los espectros}

Se obtuvieron los espectros de absorción mediante escaneo NIRS (400-2500 nm, intevalo $2 \mathrm{~nm}$ ) en modo de reflectancia de las 75 muestras. Los datos se guardaron como el logaritmo (1/R) (donde $\mathrm{R}=$ reflectancia). Para la calibración, se seleccionaron 50 espectros representativos mediante muestreo aleatorio (Alomar y Fuchslocher, 1998). La validación externa se realizó usando los 25 espectros restantes. El rango espectral de las bandas de absorción NIRS de los constituyentes nutricionales de rye grass italiano se encuentra entre 1100 y $2400 \mathrm{~nm}$ (Wehling, 2008).

Desarrollo de la calibración NIRS y análisis de regresión

El tratamiento matemático para la calibración y validación fue mediante cuadrados mínimos parciales (PLS) Derivada 1, según Marten (1983). La ecuación básica de calibración (Vásquez et al., 2004) está dada por $Y=a+b_{1} X_{1}+b_{2} X_{2}+\ldots . .+b_{n} X_{n}$, donde, $\mathrm{Y}=$ componente a ser calibrado, $\mathrm{X}_{1} \mathrm{X}_{2} \ldots \mathrm{X}_{\mathrm{n}}$ $=\mathrm{n}$ variables espectrales independientes, $\mathrm{b}_{1}$ $b_{2} \ldots . . b_{n}=n$ coeficientes de regresión, y a $=$ el intercepto.

Para la verificación se utilizó un análisis de regresión para establecer la exactitud de los valores obtenidos mediante método químico de referencia de proteína cruda (PC), extracto etéreo (EE), cenizas totales $(\mathrm{CZ})$, fibra cruda (FC) y fibra detergente neutro (FDN) con las ecuaciones del NIRS.

Se realizó estadística descriptiva, determinando la media, desviación estándar, valor máximo y mínimo, y el coeficiente de variación de los parámetros químico nutricionales del pasto.

Como parámetro de exactitud para la evaluación del modelo de calibración y estimar su capacidad predictiva, se utilizó el co- eficiente de correlación ( $R$ ), el coeficiente de determinación [ $\mathrm{R}^{2}$, Ec. (1)], RMSEC [Ec. (2)]: error de predicción en calibración para el conjunto de calibración; RMSEP [Ec. (3)]: error de predicción en validación externa para el conjunto de validación; RPD [Ec. (4)]: relación entre la desviación estándar del método químico de referencia y el error de predicción encontrado en el modelo NIRS; y RER [Ec. (5)]: proporción de rango de datos con el error de predicción (Cozzolino, 2002; Magwaza et al., 2016).
(1) $R^{2}=1-\frac{\sum\left(\text { Ycal }-Y_{\text {mean }}\right)^{2}}{\sum(\text { Ycal }- \text { Yact })^{2}}$
(2) $\mathrm{RMSEC}=\sqrt{\sum(Y c a l-Y a c t)^{2} / n}$
(3) RMSEP $=\sqrt{\sum(\text { pred }- \text { Yact })^{2} / n}$
(4) RPD = SD / RMSEP
(5) RPD = rango / RMSEP

donde $\mathrm{n}=$ número de espectro; $\mathrm{Y}_{a c t}=$ valor actual; $\mathrm{Y}_{\text {mean }}=$ valor promedio; $\mathrm{Y}_{\text {cal }}=$ valor calculado; $\mathrm{Y}_{\text {pred }}=$ valor de predicción del atributo; y SD = desviación estándar de los valores referenciales.

Según Vymazal (2008), el modelo debe tener alto $\mathrm{R} y \mathrm{R}^{2}$, bajo RMSEC y RMSEP, RPD superior a 3 y RER superior a 10 .

\section{Resultados y Discusión}

\section{Análisis Proximal}

Los resultados de los análisis de laboratorio para los colectivos de calibración y validación externa se presentan en los cuadros 1 y 2, respectivamente. En ambos casos, los valores de PC, EE, CZ, FC y FDN presentaron un amplio rango de variabilidad, favorable para el desarrollo de ecuaciones de calibración NIRS. Asimismo, reflejan la variación en el contenido de nutrientes según la edad de crecimiento del pasto. 
Cuadro 1. Análisis químico proximal y fibra detergente neutro - FDN (\%) de rye grass italiano (Lolium multiflorum Lam) y variación de la población para la calibración del NIRS de cada constituyente ( $\mathrm{n}=50$ muestras)

\begin{tabular}{lccccc}
\hline Componente & $\begin{array}{c}\text { Proteína } \\
\text { cruda }\end{array}$ & $\begin{array}{c}\text { Extracto } \\
\text { etéreo }\end{array}$ & $\begin{array}{c}\text { Cenizas } \\
\text { totales }\end{array}$ & Fibra cruda & FDN \\
\hline Rango & $8.53-$ & $1.00-$ & $8.10-$ & $14.30-$ & $52.0-$ \\
& 24.19 & 6.70 & 15.78 & 21.72 & 66.5 \\
Promedio & 19.02 & 4.53 & 12.79 & 16.50 & 60.98 \\
$\begin{array}{l}\text { Desviación } \\
\text { estándar }\end{array}$ & 5.03 & 1.20 & 1.94 & 2.49 & 3.09 \\
$\begin{array}{l}\text { Coeficiente de } \\
\text { variación }\end{array}$ & 0.26 & 0.26 & 0.15 & 0.15 & 0.05 \\
Varianza & 25.26 & 1.43 & 3.75 & 6.20 & 9.55 \\
\hline
\end{tabular}

Cuadro 2. Análisis químico proximal y fibra detergente neutro - FDN (\%) de rye grass italiano (Lolium multiflorum Lam) y variación de la población para la validación de cada constituyente ( $\mathrm{n}=25$ muestras)

\begin{tabular}{lccccc}
\hline Componente & $\begin{array}{c}\text { Proteína } \\
\text { cruda }\end{array}$ & $\begin{array}{c}\text { Extracto } \\
\text { etéreo }\end{array}$ & $\begin{array}{c}\text { Cenizas } \\
\text { totales }\end{array}$ & Fibra cruda & FDN \\
\hline Rango & $8.51-$ & $1.00-$ & $8.11-$ & $13.98-$ & $52.6-$ \\
Promedio & 24.31 & 7.48 & 14.75 & 20.97 & 65.2 \\
$\begin{array}{l}\text { Desviación } \\
\text { estándar }\end{array}$ & 19.72 & 4.45 & 12.41 & 15.83 & 60.34 \\
$\begin{array}{l}\text { Coeficiente de } \\
\text { variación }\end{array}$ & 0.64 & 1.42 & 1.79 & 1.96 & 3.42 \\
Varianza & 21.57 & 0.32 & 0.14 & 0.12 & 0.05 \\
\hline
\end{tabular}

La PC presentó un valor de $19.02 \%$ en el colectivo de calibración y de $19.72 \%$ en el colectivo de validación externa, valores por encima del promedio dado por Laforé et al. (1999) de $9.60 \%$ y de $14.7 \%$ reportado por Flores et al. (2013) para rye grass italiano cultivado en el valle del Mantaro, HuancayoPerú y en la zona costera de Galicia, España, respectivamente. Por otro lado, similar al $18.8 \%$ hallado por Posada et al. (2013) en Medellín, Colombia, a 2750 msnm.

El promedio en EE fue de $4.53 \%$ en el colectivo de calibración y de $4.45 \%$ en el de validación externa. Estos valores fueron superiores al 2.61 reportado por Villalobos y 
Sánchez (2010) en rye grass (Lolium multiflorum Lam), cultivado en zonas altas de Costa Rica, pero similar al $4.7 \%$ hallado por Posada et al. (2013) en rye grass italiano.

El valor de las CZ fue de 12.79 y $12.41 \%$ en los colectivos calibración y validación, respectivamente, mayor al $9.96 \%$ obtenido por Villalobos y Sánchez (2010) en rye grass. Asimismo, el promedio de FC fue de $16.50 \%$ en colectivo de calibración y $15.83 \%$ en colectivo de validación externa; superiores a lo hallado por Villalobos y Sánchez (2010) en rye grass $(15.40 \%)$; pero inferiores a lo reportado por Posada et al. (2013) en rye grass italiano $(23.90 \%)$. Los resultados de FDN tuvieron un promedio de $60.98 \%$ y $60.34 \%$ en los colectivos calibración y validación y fueron superiores al $51 \%$ hallado por Laforé et al (1999), al 49.76\% de Villalobos y Sánchez (2010) y al 40.8\% hallado por Posada et al. (2013).

Los resultados encontrados reflejan la amplia variación existente en la composición nutricional del rye grass italiano cultivado bajo las condiciones climáticas y características del suelo del valle del Mantaro a $3300 \mathrm{msnm}$; factores que tienen gran influencia en la composición nutritiva y calidad de los pastos en esta región (Bojórquez et al., 2015).

\section{Calibración y Validación Externa}

La Figura 1 muestra el conjunto de datos espectrales del colectivo de muestras utilizadas en el trabajo. Se observa algunas irregularidades en un determinado número de muestras en la región de sobretonos de longitud de onda 2050 a 2150 , pero conservando su característico «paralelismo» con relación al colectivo global, lo que permite garantizar la exactitud de predicción del contenido nutricional en el rango de calibración.

En el Cuadro 3 se muestran los estadísticos determinados por las mejores ecuaciones de calibración y validación externa de las muestras para los constituyentes PC, EE, CZ, FC y FDN. Para R y R ${ }^{2}$ se tuvo en cuenta el criterio de Shenk et al. (1992) y Andrés et al. (2005), quienes señalan que valores de $0.50-0.69,0.70-0.89 \mathrm{y}>0.90$ indican una calibración ineficaz, regular, y buena, respectivamente, considerando si el modelo discrimina muestras de alto y bajo contenido en el parámetro analizado.

Los resultados de calibración muestran valores altos de $\mathrm{R} \mathrm{y}^{2}(>0.90)$ para la mayoría de constituyentes; sin embargo, se halló un valor de $\mathrm{R}^{2}=0.81$ para para el constituyente $\mathrm{FC}$, lo que indicaría una calibración regular para este parámetro. Cozzolino (2002), analizando insumos para animales mediante NIRS, reportó valores de $\mathrm{R}^{2}$ de 0.98 para $\mathrm{PC}$, 0.93 para $\mathrm{FC}$ y 0.92 para $\mathrm{CZ}$; en tanto que Keim et al. (2015) obtuvieron valores de $\mathrm{R}^{2}$ de 0.99 para PC y 0.95 para FDN en un estudio de determinación de estos constituyentes en pasturas incubadas en rumen.

Los valores de RMSEC para los constituyentes de rye grass italiano fueron bajos, observándose los mejores resultados para los constituyentes EE (0.29) y CZ (0.57). Asekova et al. (2016) obtuvieron valores de 0.608 y 0.387 de RMSEC para PC y EE, respectivamente, en granos de soya. Asimismo, Magwaza et al. (2016) reportaron valores de $0.26\left(\mathrm{R}^{2}=0.98\right)$ para 104 variedades de camote; Vásquez et al. (2004) reportaron valores de $0.26\left(\mathrm{R}^{2}=0.99\right)$ para $\mathrm{PC}, 0.09\left(\mathrm{R}^{2}=0.93\right)$ para $\mathrm{EE}$; $0.21\left(\mathrm{R}^{2}=0.99\right)$ para $\mathrm{CZ}$ y 0.69 $\left(\mathrm{R}^{2}=0.95\right)$ para $\mathrm{FDN}$, en 70 muestras de 18 variedades de pasto guinea en Colombia. Estos reportes presentan valores $\mathrm{R}^{2}$ similares a los obtenidos en el presente estudio para rye grass italiano. Por otro lado, Castro et al. (2001) reportaron valores de RMSEC de 0.63 $\left(\mathrm{R}^{2}=0.98\right)$ para $\mathrm{PC}$ y $1.97\left(\mathrm{R}^{2}=0.94\right)$ para FDN al analizar espectros de rye grass inglés, dáctilo y praderas mixtas.

Los resultados del modelo de validación externa, indicados en el Cuadro 3, muestran altos coeficientes de correlación (R), $0.97 \mathrm{y}$ 0.93 , y regulares coeficientes de determinación $\left(\mathrm{R}^{2}\right), 0.94$ y 0.86 , para los constituyentes PC y FDN, respectivamente. El valor más 


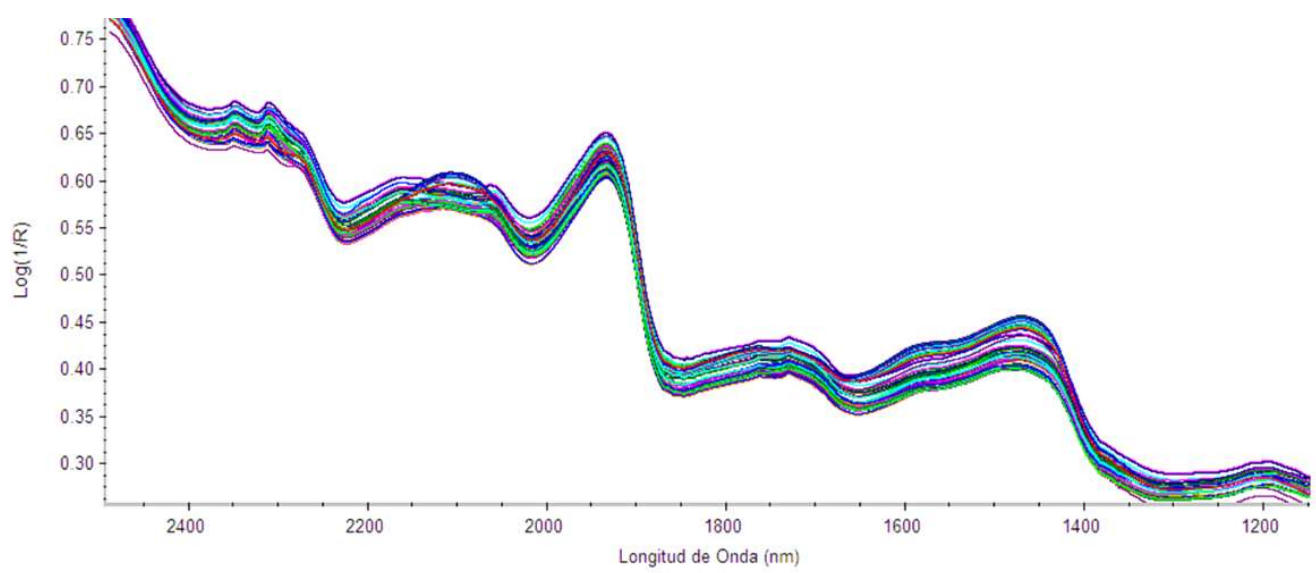

Figura 1. Espectro NIRS en $\log 1 / \mathrm{R}$ y longitud de onda de las muestras de rye grass italiano (Lolium multiflorum Lam) ( $\mathrm{n}=75$ muestras). Laboratorio de Bioquímica, Nutrición y Alimentación Animal (LBNAA), Facultad de Medicina Veterinaria, Universidad Nacional Mayor de San Marcos, Lima

Cuadro 3. Estadísticos de calibración y validación de las ecuaciones obtenidas para la determinación de análisis proximal y fibra detergente neutro (FDN) de Rye grass italiano (Lolium multiflorum Lam)

\begin{tabular}{lcccccccc}
\hline \multirow{2}{*}{ Constituyente } & \multicolumn{4}{c}{ Calibración } & \multicolumn{5}{c}{ Validación } \\
\cline { 2 - 9 } & $\mathrm{n}$ & $\mathrm{R}$ & $\mathrm{R}^{2}$ & $\mathrm{RMSEC}^{\mathrm{a}}$ & $\mathrm{n}$ & $\mathrm{R}$ & $\mathrm{R}^{2}$ & RMSEP $^{\mathrm{b}}$ \\
\hline Proteína cruda & 50 & 0.98 & 0.96 & 1.02 & 25 & 0.97 & 0.94 & 1.19 \\
Extracto etéreo & 50 & 0.97 & 0.94 & 0.29 & 25 & 0.84 & 0.71 & 1.05 \\
Cenizas totales & 50 & 0.95 & 0.90 & 0.57 & 25 & 0.86 & 0.74 & 0.92 \\
Fibra cruda & 50 & 0.90 & 0.81 & 1.07 & 25 & 0.84 & 0.71 & 1.13 \\
FDN & 50 & 0.95 & 0.90 & 1.01 & 25 & 0.93 & 0.86 & 1.25 \\
\hline
\end{tabular}

$\mathrm{R}$ : Coeficiente de correlación; $\mathrm{R}^{2}$ : Coeficiente de determinación

a Raíz cuadrada media del error de calibración

${ }^{\text {b }}$ Raíz cuadrada media del error de predicción

bajo de RMSEP se muestra en el constituyente CZ (0.92) y el valor más alto en FDN (1.25).

El estadístico RPD (Residual Predictive Deviation), también utilizado para evaluación de ecuaciones NIRS en relación a precisión y exactitud, mide la relación entre la desviación estándar (SD) del análisis químico y el RMSEP del constituyente. Si esta relación es menor a 3, las ecuaciones obtenidas tienen un bajo poder de predicción (Murray, 1993). En el Cuadro 4 se presentan los resultados de RPD superiores a 3 en los constituyentes PC, EE, CZ y FDN, obteniéndose el mayor valor para EE (4.90), seguido de PC (4.55). El constituyente FC resultó con un RPD inferior a 3, lo que significaría que la calibración obtenida con el tratamiento matemático empleado en este estu- 
Cuadro 4. Estadísticos estimadores de la calidad de la calibración para análisis proximal y fibra detergente neutro de rye grass italiano (Lolium multiflorum Lam)

\begin{tabular}{lccc}
\hline & \multicolumn{3}{c}{ Estadístico $^{1}$} \\
\cline { 2 - 4 } Constituyente & $\begin{array}{c}\text { TM } \\
(\mathrm{a}, \mathrm{b}, \mathrm{c})\end{array}$ & RER & RPD \\
\hline Proteína cruda & $1,5,5$ & 15.49 & 4.55 \\
Extracto etéreo & $1,5,5$ & 22.34 & 4.90 \\
Cenizas totales & $1,5,5$ & 11.65 & 3.14 \\
Fibra cruda & $1,5,5$ & 6.53 & 1.83 \\
Fibra detergente neutro & $1,5,5$ & 12.47 & 3.39 \\
(FDN) & & & \\
\hline
\end{tabular}

1 TM: Tratamiento matemático (a: Derivada; b: Gap; c: Smooth). RER: Range Error Ratio (Proporción de rango de datos con el error). RPD: Residual Predictive Deviation (Proporción de la desviación con el error)

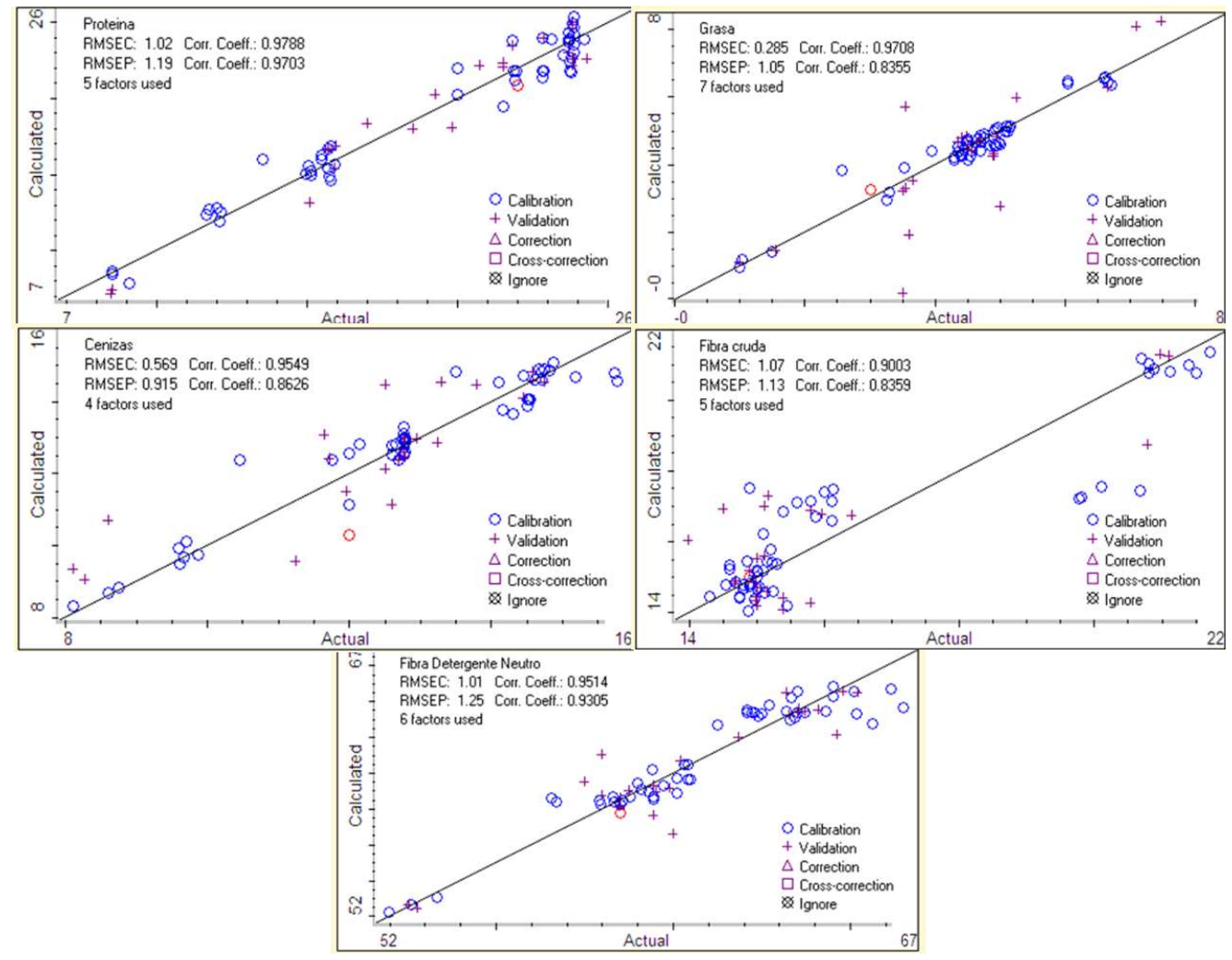

Figura 2. Curvas de calibración y validación obtenidas para los constituyentes proteína cruda, extracto etéreo, cenizas totales, fibra cruda y fibra detergente neutro 
dio no estaría prediciendo valores químico proximales correctos, que sería consecuencia de la variabilidad natural del material biológico experimental.

Williams (2001) señala que resultados del RPD $>3$ permiten el uso de la calibración obtenida para clasificación y evaluación de muestras en laboratorio. El estadístico RER (Range Error Ratio) se emplea para evaluar el rendimiento del modelo de calibración de tal forma que si RER $\geq 4$, la calibración es aceptable para análisis cualitativo de muestras; si RER $\geq 10$, la calibración es aceptable para control de calidad; y si RER $\geq 15$, la calibración es óptima para cuantificación (AACC, 2016). De la correlación entre la señal espectral del barrido y el método químico (calibración) se obtuvo el modelo matemático estadístico. La totalidad de ecuaciones fueron desarrolladas con la primera deriva$\mathrm{da}$, una distancia entre los puntos de longitudes de onda (Gap) igual a 5 y una eliminación de ruido (Smoth) Savitzky-Golay de 5 en el orden polinomial 3 .

En la Figura 2 se observa una alta correlación y tendencia lineal entre los valores calculados mediante NIRS (calculated) y químico proximal del LBNAA (actual) en los colectivos de calibración y validación para los constituyentes PC, EE, CZ, FC y FDN.

La exactitud de las predicciones NIRS dependerá de la precisión con que se realicen los análisis químicos y los tratamientos matemáticos. Los altos valores obtenidos de RPD (>3) y RER (>10) indican una capacidad de predicción alta para el contenido de PC, EE, CZ y FDN en muestras de rye grass italiano (Lolium multiflorum Lam) mediante la técnica NIRS.

\section{Conclusiones}

- Las ecuaciones de calibración y validación NIRS obtenidas permiten una óptima predicción cuantitativa de proteína cruda, extracto etéreo, cenizas totales, fibra cruda y fibra detergente neutro en rye grass italiano (Lolium multiflorum Lam).

- Para el constituyente fibra cruda, la calibración NIRS obtenida muestra una baja predicción cuantitativa.

- El análisis proximal de rye grass italiano (Lolium multiflorum Lam) presentó valores promedio de $19.02 \%$ de proteína cruda, $4.53 \%$ para extracto etéreo, $12.79 \%$ para cenizas, $16.50 \%$ para fibra cruda y $60.98 \%$ para fibra detergente neutro.

\section{Literatura Citada}

1. Alomar D, Fuchslocher R. 1998. Fundamentos de la espectroscopía de reflectancia en el infrarrojo cercano (NIRS) como método de análisis de forraje. Agro Sur 26(1): 88-104.

2. Andrés L, Murray I, Calleja A, Giráldez FJ. 2005. Review: nutritive evaluation of forages by near infrared reflectance spectroscopy. J Near Infrared Spec 13:301-311. doi: 10.1255/ jnirs.561

3. Asekova S, Han S, Choi H, Park S, Shin D, Kwon C, Shannon J, Lee J. 2016. Determination of forage quality by near-infrared reflectance spectroscopy in soybean. Turk J Agric For 40: 45-52. doi: 10.3906/tar-1407-33

4. Andueza D, Picard F, Jestin M, Andrieu J, Baumont R. 2011. NIRS prediction of the feed value of temperate forages: efficacy of four calibration strategies. Animal 5: 1002-1013. doi: 10.1017/S1751731110002697

5. [AOAC] Association of Official Analytical Chemists. 1990. Official methods of analysis. $15^{\text {th }}$ ed. Vol 1 . Arlington, USA: AOAC. 746 p.

6. Bojórquez CL, Rojas JD, Ordóñez JH. 2015. Pastos cultivados en el valle del Mantaro. Lima: Fondo Editorial UNMSM. 147 p. 
7. CARE Ancash-Perú. 2011. Cultivando pastos asociados: sistematización de la experiencia. Proyecto de Cadenas Productivas Alli Allpa. Lima: Fondo Minero Antamina. $35 \mathrm{p}$.

8. Castro P, Fernández B, Valladares J. 2001. Análisis de pastos mediante NIRS. En: XLV Reunión Científica de la SEEP). España: SEEP.

9. Cozzolino D, Fassio A, Gimenez A. 2003. The use of near infrared reflectance spectroscopy (NIRS) to predict the composition of whole maize plants. J Sci Food Agric 81: 142-146.

10. Cozzolino D. 2002. Uso de espectroscopía de reflectancia en el infrarrojo cercano (NIRS) para análisis de alimentos para animales. Agrociencia 6: 25-32.

11. Dambergs R, Cozzolino D, Cynkar W, Janik L, Gishen M. 2006. The determination of red grape quality parameters using the Local algorithm. J Near Infrared Spectrosc 14: 71-79.

12. Decruyenaere $V$, Planchon $V$, Dardenne P, Stilmant D. 2015. Prediction error and repeatability of near infrared reflectance spectroscopy applied to faeces samples in order to predict voluntary intake and digestibility of forages by ruminants. Anim Feed Sci Tech 205: 49-59.

13. Decruyenaere V, Lecomte $P$, Demarquilly C, Aufrere J, Dardenne P, Stilmant D, Buldgen A. 2009. Evaluation of green forage intake and digestibility in ruminants using near infrared reflectance spectroscopy (NIRS): developing a global calibration. Anim Feed Sci Tech 148: 138-156. doi: 10.1016/j.anifeedsci.2008.03.007

14. [FAO] Food and Agriculture Organization of the United Nations. 2011. Quality assurance for animal feed analysis laboratories. FAO Animal Production and Health Manual 4. Rome: FAO. $178 \mathrm{p}$.

15. Flores G, Díaz N, Díaz M, Valladares J, Pereira S, Fernández BL, Resch C, et al. 2013. Evaluación de métodos de laboratorio para la predicción de la digestibilidad in vivo de la materia orgánica de ensilajes de hierba y planta entera de maíz. Pastos 43(1): 5-99.

16. García-Ciudad A, García-Criado B, Pérez-Corona M, Vázquez de Aldana B, Ruano-Ramos A. 1993. Application of near-infrared reflectance spectroscopy to chemical analysis of heterogeneous and botanically complex grassland samples. J Sci Food Agric 63: 419-426. doi: 10.1002/jsfa.2740630407

17. Givens DI, De Boever J, Deaviee E. 1997. The principles, practices and some future applications of near infrared spectroscopy for predicting the nutritive value of foods for animals and humans. Nutr Res Rev 10: 83-114. doi: 10.1079/ NRR19970006

18. [INEI] Instituto Nacional de Estadística e Informática. 2012. IV Censo Nacional Agropecuario: Resultados Definitivos. Lima, Perú: INEI-Ministerio de Agricultura y Riego. $62 \mathrm{p}$.

19. Keim A, Charles B, Alomar A. 2015. Prediction of crude protein and neutral detergent fibre concentration in residues of in situ ruminal degradation of pasture samples by near-infrared spectroscopy (NIRS). Anim Prod Sci 56: 1504-1511. doi: 10.1071/AN14822

20. Krähmer A, Gudi G, Weiher N, Gierus M, Schütze W, Schulz H. 2013. Characterization and quantification of secondary metabolite profiles in leaves of red and white clover species by NIR and ATR-IR spectroscopy. Vib Spectrosc 68: 96-103. doi: 10.1016/j.vibspec.2013.05.012

21. Laforé M, San Martín F, Custodio R, Arbaiza T, Carcelén F. 1999. Diagnóstico alimenticio y composición químico nutricional de los principales insumos de uso pecuario del valle del Mantaro. Rev Inv Vet Peru 10: 74-78. doi: 10.15381/ rivep.v10i2.6760

22. Lascano CE. 2002. Caracterización de pasturas para maximizar la producción animal. Arch Latinoam Prod Anim 10: 126-132. 
23. Magwaza L, Messo Naidoo S, Laurie $S$, Laing M, Shimelis H. 2016. Development of NIRS models for rapid quantification of protein content in sweetpotato [Ipomoea batatas (L.) LAM.]. Food Scie Tech 72: 63-70. doi: 10.1016/j.lwt.2016.04.032

24. Marten G, Halgerson J, Cherney J. 1983. Quality prediction of small grain forages by near infrared reflectance spectroscopy. Crop Sci 23: 94-101. doi: 10.2135/cropsci1983.-0011183X$002300010027 \mathrm{x}$

25. Murray I. 1993. Forage analysis by near infrared reflectance spectroscopy. In: Davies A, Baker R, Grant S, Laidlaw A (eds). Sward measurement handbook, $2^{\text {nd }}$ ed. UK: British Grassland Soc. p 285-312.

26. Ordoñez J, Bojórquez, C. 2004. Establecimiento del Lolium multiflorum con cinco densidades sobre pasturas degradadas como una alternativa a la siembra de cultivos agrícolas. Rev Inv Vet Perú 15: 87-91. doi: 10.15381/rivep.v15i2.1569

27. Posada S, Cerón J, Arenas J, Hamedt J, Alvarez A. 2013. Evaluación del establecimiento de ryegrass (Lolium $\mathrm{sp}$ ) en potreros de kikuyo (Pennisetum clandestinum) usando la metodología de cero labranza. Ces Med Vet Zootec 8(1): 23-32.

28. Pereira S, Valladares J, Flores G, Díaz $N$, Fernández B, Resch C, Gonzales $A$, et al. 2012. Rendimiento y valor nutritivo de nuevas leguminosas anuales como cultivo de invierno en rotaciones forrajeras intensivas en Galicia. Pastos 42(1): 29-50.

29. Shenk J, Westerhaus M. 1994. The application of near infrared reflectance spectroscopy (NIRS) to forage analysis. In: Fahey GC, Collins M, Mertens DR, Moser LE (eds). Forage quality, evaluation and utilization. Madison, Wisconsin, USA: American Society of Agronomy. p 406-446.

30. Shenk J, Workman J, Westerhaus M. 1992. Application of NIR spectroscopy to agricultural products. In: Burns DA, Ciurczak EW (eds). Handbook of nearinfrared analysis. New York, USA: CRC Press. p 383-431.

31. Tellado S, Orpez R, Muñoz-Cobo J, Azorit C. 2015. Fecal-FT-NIRS as a noninvasive tool for assessing diet quality of Mediterranean deer. Rangeland Ecol Manage 68: 92-99. doi: 10.1016/ j.rama.2014.12.003

32. Vásquez D, Abadia B, Arreaza L. 2004. Aplicación de la espectroscopía de reflectancia en el infrarrojo cercano (NIRS) para la caracterización nutricional del pasto guinea y del grano de maíz. Rev Corpoica 5: 49-55.

33. Van Soest PJ, Roberts JB, Lewis BA. 1991. Methods for dietary fiber, neutral detergent fiber, and nonstarch polysaccharides in relation to animal nutrition. $\mathrm{J}$ Dairy Sci 74: 3583-3597. doi: 10.3168/ jds.S0022-0302(91)78551-2

34. Villalobos L, Sánchez J. 2010. Evaluación agronómica y nutricional del pasto ryegrass perenne tetraploide (Lolium perenne) producido en lecherías de las zonas altas de Costa Rica. I. Producción de biomasa y fenología. Agronomía Costarricense 34(1): 31-42.

35. Vymazal J. 2008. Wastewater treatment, plant dynamics and management in constructed and natural wetlands. Czech Republic: Springer. $348 \mathrm{p}$.

36. Wehling R. 2008. La espectroscopia infrarroja. En: Nielsen S (ed). Análisis de los alimentos. España: Acribia. p 453467.

37. Williams P. 2001. Implementation of near-infrared tecnology. In: Williams PC, Norris KH (eds). St. Paul, MN, USA: American Association of Cereal Chemist. p 145-169.

38. Woolnough AP, Foley WJ. 2002. Rapid evaluation of pasture quality for a critically endangered mammal, the northern hairy-nosed wombat (Lasiorhinus krefftii). Wildlife Res 29: 91-100. doi: 10.1071/WR00050 